\title{
Ethical issues in biomedical research using electronic health records: a systematic review
}

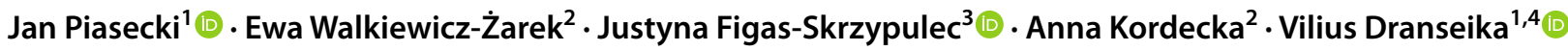

Accepted: 9 June 2021 / Published online: 19 June 2021

(c) The Author(s) 2021

\begin{abstract}
Digitization of a health record changes its accessibility. An electronic health record (EHR) can be accessed by multiple authorized users. Health information from EHRs contributes to learning healthcare systems' development. The objective of this systematic review is to answer a question: What are ethical issues concerning research using EHRs in the literature? We searched Medline Ovid, Embase and Scopus for publications concerning ethical issues of research use of EHRs. We employed the constant comparative method to retrieve common ethical themes. We descriptively summarized empirical studies. The study reveals the breadth, depth, and complexity of ethical problems associated with research use of EHRs. The central ethical question that emerges from the review is how to manage access to EHRs. Managing accessibility consists of interconnected and overlapping issues: streamlining research access to EHRs, minimizing risk, engaging and educating patients, as well as ensuring trustworthy governance of EHR data. Most of the ethical problems concerning EHR-based research arise from rapid cultural change. The framing of concepts of privacy, as well as individual and public dimensions of beneficence, are changing. We are currently living in the middle of this transition period. Human emotions and mental habits, as well as laws, are lagging behind technological developments. In the medical tradition, individual patient's health has always been in the center. Transformation of healthcare care, its digitalization, seems to have some impacts on our perspective of health care ethics, research ethics and public health ethics.
\end{abstract}

Keywords Electronic health records $\cdot$ Learning healthcare system $\cdot$ Embedded research $\cdot$ Ethics $\cdot$ consent $\cdot$ Systematic review

\section{Abbreviations \\ EHR Electronic health records \\ LHS Learning healthcare system \\ CCM Constant comparative method}

Jan Piasecki

jan.piasecki@uj.edu.pl

1 Department of Philosophy and Bioethics, Faculty of Health Sciences, Medical College, Jagiellonian University, Michalowskiego 12, 31-126 Krakow, Poland

2 HTA Registry Sp. z o.o. Sp. K, Herzoga 15, 30-252 Krakow, Poland

3 Fundacja Optimum Pareto (Optimum Pareto Foundation), ul. Celna 6/9, 30-507 Krakow, Poland

4 Institute of Philosophy, Vilnius University, 9/1 Universiteto, 01513 Vilnius, Lithuania

\section{Introduction}

An electronic health record (EHR) is a technological innovation that consists in digitization of an individual patient's health information. EHRs have already changed the landscape of biomedical research (Häyrinen et al. 2008; Foley and Fairmichael 2015; Evans 2016). The digitization of a paper-based health record alters its accessibility. A paper health record can be accessed only physically in the hospital archives, whereas an EHR can be accessed electronically by multiple authorized users from remote locations (Häyrinen et al. 2008; Evans 2016). Easy, speedy, and relatively cheap access to health information is the main fuel of any learning healthcare system (LHS) (Evans 2016). In an LHS, a process of generating scientific knowledge is embedded in practice: health information produced in the course of providing and receiving healthcare, is collected and analyzed; and then the subsequently generated knowledge is applied to current practice; the cycle starts again (Friedman and Macy 2014). 
However, secondary use of an EHR beyond the scope of clinical care raises a series of ethical questions.

These ethical questions concern the necessity of requirement of informed consent (Helgesson and Eriksson 2008; Hansson 2010), and the limits of physician-patient confidentiality in the context of embedded research and risk of being re-identified (Sweeney 2000; El Emam et al. 2011, 2015; Simon et al. 2019). Data extracted from an individual EHR, if leaked, can be potentially used to deny health services, insurance, and bank products, as well as to stigmatize individuals and groups. However, our review shows also that embedded research poses ethical challenges for healthcare professionals and healthcare institutions who are not always comfortable with sharing health data for the purpose of research, deeming that it could undermine relationships with their patients and their reputation (Simon et al. 2017). Moreover, EHRs can be also considered as an instrument of patients' empowerment and instrument of patients' contribution to progress in medicine and protection of public health.

Laws and guidelines regulating the collection and processing of personal and health information can differ from country to country, but most developed economies have extensive regulations concerning data. However, ethical and practical problems seem persistent despite the existing numerous laws. In the US, the Ascension and Google's Project Nightingale sparked public outrage (Pilkington 2019). In the UK, the care.data project faced vigorous public resistance (Anderson 2015; Hall 2016), and reportedly a similar project in Denmark also was an issue of controversy (Skovgaard et al. 2019). In all these cases, research activities, even though they were, strictly speaking, legal, were rejected by the public and became politically infeasible. These examples demonstrate that following the laws is not always sufficient for ethical action.

Moreover, legislation does not fully keep pace with technological development and private sector activities (Aicardi et al. 2016; Rumbold and Pierscionek 2017; Chassang 2017; Cohen and Mello 2018). In addition, individuals are not aware of existing safeguards (Hill et al. 2013). Therefore, it seems there is a need for ethical clarity and consensus among policy makers, healthcare providers, software developers, researchers, and patients in regard to ethical standards for research use of EHRs. Building an ethical and conceptual framework for trustworthy LHSs powered by EHRs is still ahead (Evans 2016).

The regulatory effort should be preceded by an impartial, maximally transparent and comprehensive process of evidence gathering. A systematic review of literature helps to meet these standards, providing decision-makers with a spectrum of ethical challenges that are currently discussed and should be taken into consideration (Klingler et al. 2017).

The ethical issues of EHRs in the context of biomedical research have not as yet been the subject of systematic literature review. However, the ethical problems concerning related questions, such as research in digital databases (Aitken et al. 2016), public health surveillance (Klingler et al. 2017), LHS (McLennan et al. 2018), ownership of health data (Mirchev et al. 2020), and public attitudes towards EHRs (Hill et al. 2013, Skovgaard et al. 2019), have been recently reviewed in a systematic manner. Our review is intended to fill this gap and answer the question: What ethical issues concerning EHRs in the context of biomedical research are discussed in the literature? We hope that our results can be useful for professionals working under various legal regimes, concerning research involving humans, privacy protection and data processing. The results of this literature review can be a point of departure in a search for practical policy solutions. Regulators, software developers, electronic security specialists and researchers who are involved in designing policies and laws for healthcare systems may use it to determine if their policies cover all ethical aspects of the EHRs present in the literature. Moreover, this review can also be informative for institutional and individual healthcare providers who struggle with policies, procedures and day-to-day decisions concerning access and sharing of patients' EHRs by giving them an exhaustive summary of ethical issues that should be addressed. We hope this review of literature can be a starting point for further normative analyses and research, especially into problems that have not been sufficiently discussed in the literature but can be important for the development of a trustworthy LHS.

\section{Methods}

The protocol of the review was registered on Prospero in advance (CRD42018094526) and we followed the PRISMA protocol as far as it is applicable to a qualitative review: we did not follow the recommendations for data synthesis, and we did not conduct meta-bias assessment.

\section{Eligibility criteria}

In our analysis, we included all papers that met the conjunction of three criteria: papers that (i) discuss ethical issues concerning (ii) the use of EHRs (iii) in the context of either biomedical research, or learning healthcare systems, or quality improvement activities. We defined the term ethical issue as roughly referring to one of the four ethical principles of biomedical ethics distinguished by T. L. Beauchamp and J. Childress: the principle of respect for autonomy, the principles of beneficence, non-maleficence and justice (Beauchamp and Childress 2013). We noticed that every general principle also covers more specific ethical issues. For instance, the principle of respect for autonomy covers the principle of respect for privacy, the requirement of informed 
consent, and the obligation to restrict disclosure of health information. Therefore, we included a paper if it discussed either general or more specific ethical issues that can be subsumed under one of the four principles. However, the four principles and their derivatives were considered only as a signal for ethical issues. As we explain later, we did not limit our analysis only to those principles.

We understood the term electronic health record (EHR) as digitized health information of an individual patient which is stored electronically in a healthcare system: a single medical facility, a chain of facilities, or a national healthcare system. The terms biomedical research, learning healthcare and quality improvement are construed as activities generating generalizable knowledge in the context of healthcare. We accepted peer reviewed articles, book chapters, reports, guidelines, commentaries, and letters to the editor published in English. We excluded all papers without sufficient amount of ethical deliberation, as well as conference abstracts and newspaper articles. The term "sufficient amount of ethical deliberation" was understood as an amount of meaning that can be captured in a separate subcategory in a process of constant comparative reading. To ensure maximum objectivity of this element, two coders were involved in identifying whether inclusion criteria as described above apply to a given publication.

\section{Sources and search strategy}

We conducted systematic searches in Medline Ovid, Embase, and Scopus databases, with no time limitation on $22 / 03 / 2018$, using the subscription available at Jagiellonian University Medical College. The search strategy for each database is presented in supplementary materials (Supplement 2. Search string) and in the published protocol (Prospero CRD42018094526).

\section{Data management}

Search results were exported to an Endnote database for automated duplicate screening. EWŻ manually excluded all duplicates that were not removed during the automated screening. All records were then subsequently exported to a Microsoft Word document and a screening protocol was created. EWŻ made the Word document and the protocol available through a web-cloud to all authors involved in screening procedures. The title and abstract screening were preceded by the preliminary training phase. The aim of the training phase was ensuring that all authors and contributors understand the eligibility criteria in a uniform manner. In this phase, contributors PB and ES (under EWŻ's supervision) and JP screened the first 100 records for the eligibility criteria in order to verify the consistency of the approach used.

\section{Selection process, data collection and data analysis}

In the first phase of our review, the titles and abstracts were screened for eligibility criteria by two referees (contributors PB and ES under EWŻ and AK's supervision). All disagreements were resolved by JP. Previously undetected duplicates were excluded as well. Papers meeting the eligibility criteria were then downloaded and underwent full-text screening by JP and EWŻ. Any disagreement was resolved by discussion and consensus. The eligible papers were subsequently analyzed by JP, EWŻ, and JFS.

\section{Qualitative methodology}

We conducted qualitative analysis using the constant comparative method (CCM) that has an inductive character and consists of reading a text with an intention to capture the main recurring units of meaning (Boeije 2002; Dye et al. 2000; Gibbs 2009). When subsequent materials within the sample are analyzed, the units of meaning can be generalized and remodeled. The outcome of the entire analysis is a list of categories discerned in the papers (see: Table 1, and Supplement 1. The Full Grid). The qualitative analysis was conducted by two pairs of coders to enhance the objectivity of the process. JP created a draft grid of categories and then subsequently discussed it with EWŻ, JFS and VD. After agreeing to the final version of the grid, the authors (JP, EWŻ, and JSF) independently coded the papers.

An extraction chart of quantitative data from empirical studies was prepared by JP and consulted with JFS. Then quantitative data were independently extracted by JFS and JP in accordance with the chart. The data was then summarized in a narrative form and presented in Table 2. Narrative summaries of qualitative research were created by JP and consulted with JFS (Table 2).

\section{Possible biases and limitations}

This study has some limitations. We did not search databases other than those listed above. For example, we did not search Google Books and Google Scholar. Although the latter is well-known for containing an abundance of grey literature, a search in this database is not fully replicable (Haddaway et al. 2015, Piasecki et al. 2018). Therefore, our choice probably decreased the sample size, but at the same time it enhanced transparency and replicability of searches, which are crucial for systematic review. Moreover, the main purpose of a qualitative study is to obtain a sufficiently rich 
Table 1 Grid of categories

\begin{tabular}{|c|c|c|}
\hline Sl & Category & $\mathrm{N}$ \\
\hline \multicolumn{3}{|c|}{ A. Rationale for research using EHRs } \\
\hline 1 & Public interest & 43 \\
\hline 2 & Value of EHR research & 36 \\
\hline 3 & Justice of the healthcare system & 8 \\
\hline 4 & Private sector profits & 7 \\
\hline \multicolumn{3}{|c|}{ B. Factors affecting research use of EHRs } \\
\hline 5 & Obstructive regulations & 31 \\
\hline 6 & Regulatory facilitations and institutional support & 27 \\
\hline 7 & Technical difficulties with EHR database implementation & 25 \\
\hline 8 & $\begin{array}{l}\text { Factors hindering informing participants about research and obtaining } \\
\text { consent }\end{array}$ & 22 \\
\hline 9 & Public awareness, experience, opinions and attitudes & 24 \\
\hline 10 & Researchers' attitude & 3 \\
\hline \multicolumn{3}{|c|}{ C. Data management } \\
\hline 11 & Safety and security & 36 \\
\hline 12 & Levels of data identifiability and research & 41 \\
\hline 13 & Special types of data and their protection & 13 \\
\hline 14 & Data quality, quantity, and integrity & 26 \\
\hline 15 & Data ownership, management, and curation & 15 \\
\hline 16 & Meaningful data sharing & 19 \\
\hline 17 & Data storage, extraction, and data transfer & 30 \\
\hline 18 & Legitimacy of uses and users of EHR data & 22 \\
\hline
\end{tabular}

D. Impact of digitalization on healthcare system: providers' operations and patient engagement

19

20

21

22

23

24

E. Risks, harms and burdens of research with EHRs

25

26

27

28

29

30

31

32

33

34

35

36

37

38

39

F. Measures for subject protection

40
EHR-based research and changed professional relationships within health care institutions and between various institutions

EHR-based research and the practice-patient relationship

Medical staff and ethical responsibility in the context of EHR and EHR- 17 based research

$\begin{array}{ll}\text { Additional workload for staff and patients } & 12\end{array}$

Pivotal role of patients' contribution $\quad 18$

$\begin{array}{ll}\text { Ideas for the future: digital patient-citizenship } & 14\end{array}$

Risk to privacy 39

Compromised patients' autonomy 21

Dignitary harm—being wronged 9

Harmful or wrongful use of data $\quad 11$

Legal harm $\quad 2$

Psychological harm 3

Information risk $\quad 13$

Risk of exploitation $\quad 12$

Undue pressure to participate $\quad 2$

Undermined trust $\quad 14$

Compromised care $\quad 5$

Group mediated risks $\quad 15$

Financial conflict of interest $\quad 9$

EHRs as a challenge and potentially negative influence on healthcare $\quad 13$ practices, staff, and patient-provider communication

Risks to healthcare provider 3

\begin{tabular}{ll} 
Independent review & 26 \\
\hline
\end{tabular} 
Table 1 (continued)

\begin{tabular}{|c|c|c|}
\hline $\mathrm{S} 1$ & Category & $\overline{\mathrm{N}}$ \\
\hline 41 & Informed consent or authorization of data use & 43 \\
\hline 42 & Legal, ethical and professional regulations & 19 \\
\hline 43 & Risk assessment and risk minimization, non-maleficence & 14 \\
\hline 44 & Primary care provider consent & 2 \\
\hline 45 & Community/panel of patients consent & 2 \\
\hline 46 & Provider consent mechanism & 2 \\
\hline \multicolumn{3}{|c|}{ G. Types of consent } \\
\hline 47 & Initiation of contact & 4 \\
\hline 48 & $\begin{array}{l}\text { Options on the continuum from no consent via opt-out, to explicit and } \\
\text { informed consent }\end{array}$ & 27 \\
\hline 49 & Verbal consent, written consent & 14 \\
\hline 50 & Broad-narrow consent & 8 \\
\hline 51 & Interactive consent and granularity & 16 \\
\hline 52 & Retrospective consent & 6 \\
\hline 53 & Proxy consent & 4 \\
\hline 54 & Assent & 2 \\
\hline \multicolumn{3}{|c|}{ H. Content of consent } \\
\hline 55 & Data management: purpose, future use, storage and data sharing & 15 \\
\hline 56 & Security measures & 12 \\
\hline 57 & Benefits, risk and burdens & 10 \\
\hline 58 & Commercial application of data & 9 \\
\hline 59 & Communication of results & 4 \\
\hline 60 & Sources of research funding & 2 \\
\hline \multicolumn{3}{|c|}{ I. Reasons and motives for participation in EHRs-based research } \\
\hline 61 & Benefits of making one's EHR available & 7 \\
\hline 62 & Moral values and obligations & 13 \\
\hline 63 & $\begin{array}{l}\text { Trust in people and institutions inviting one to participate in EHR-based } \\
\text { research }\end{array}$ & 13 \\
\hline 64 & Personal cirumstances and characteristics & 14 \\
\hline \multicolumn{3}{|c|}{ J. Emotions experienced as a result of reflection on EHRs and/or participation in EHR-based research } \\
\hline 65 & $\begin{array}{l}\text { Emotions indicating respondents' positive attitude towards EHR-based } \\
\text { research }\end{array}$ & 9 \\
\hline 66 & $\begin{array}{l}\text { Emotions indicating respondents' negative attitude towards or doubts } \\
\text { about EHR-based research }\end{array}$ & 12 \\
\hline 67 & Emotional spectrum and ambivalence & 5 \\
\hline \multicolumn{3}{|c|}{ K. Ethical values, rights and obligations } \\
\hline 68 & $\begin{array}{l}\text { Autonomy, control over personal data, dignity, confidentiality and } \\
\text { privacy }\end{array}$ & 49 \\
\hline 69 & Information, public education and engagement & 22 \\
\hline 70 & Principle of beneficence & 17 \\
\hline 71 & Principle of justice & 12 \\
\hline 72 & Research integrity & 5 \\
\hline 73 & Optimal health care and clinical judgment & 3 \\
\hline 74 & Right to compensation & 1 \\
\hline
\end{tabular}

For the full Grid with the descriptions of the categories and $(\mathrm{N})$ their occurrence in the articles see Supplement 1. The Full Grid

sample and our study meets this criterion, providing us with an abundance of diverse data.

It can be argued that the four-principle ethical framework does not offer an adequate set-up for the ethical problems of EHRs in particular or that it does not offer a completely neutral approach to ethical problems in general. The former objection can be elaborated in the following way: there are new frameworks devised for the purpose of tackling ethical 












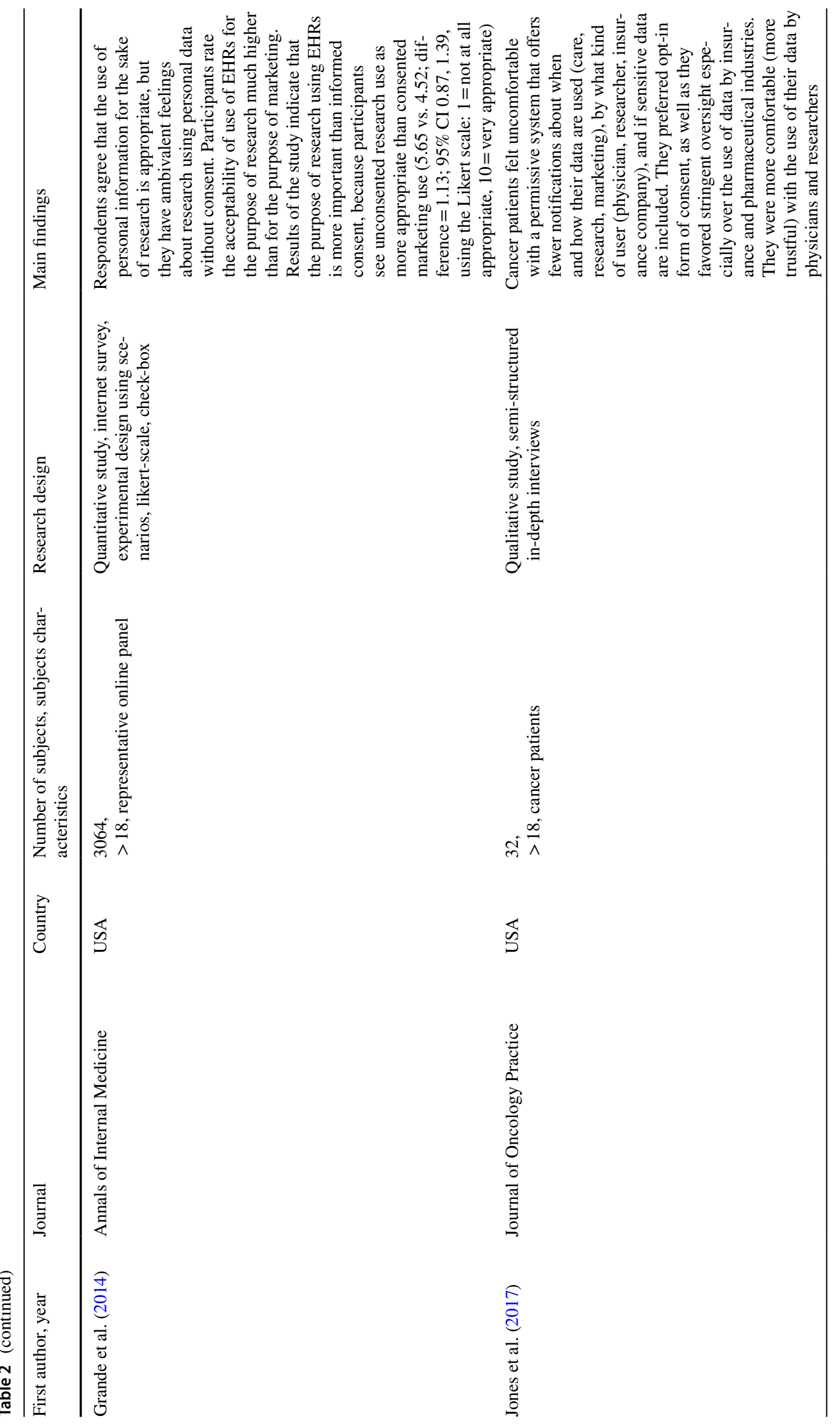




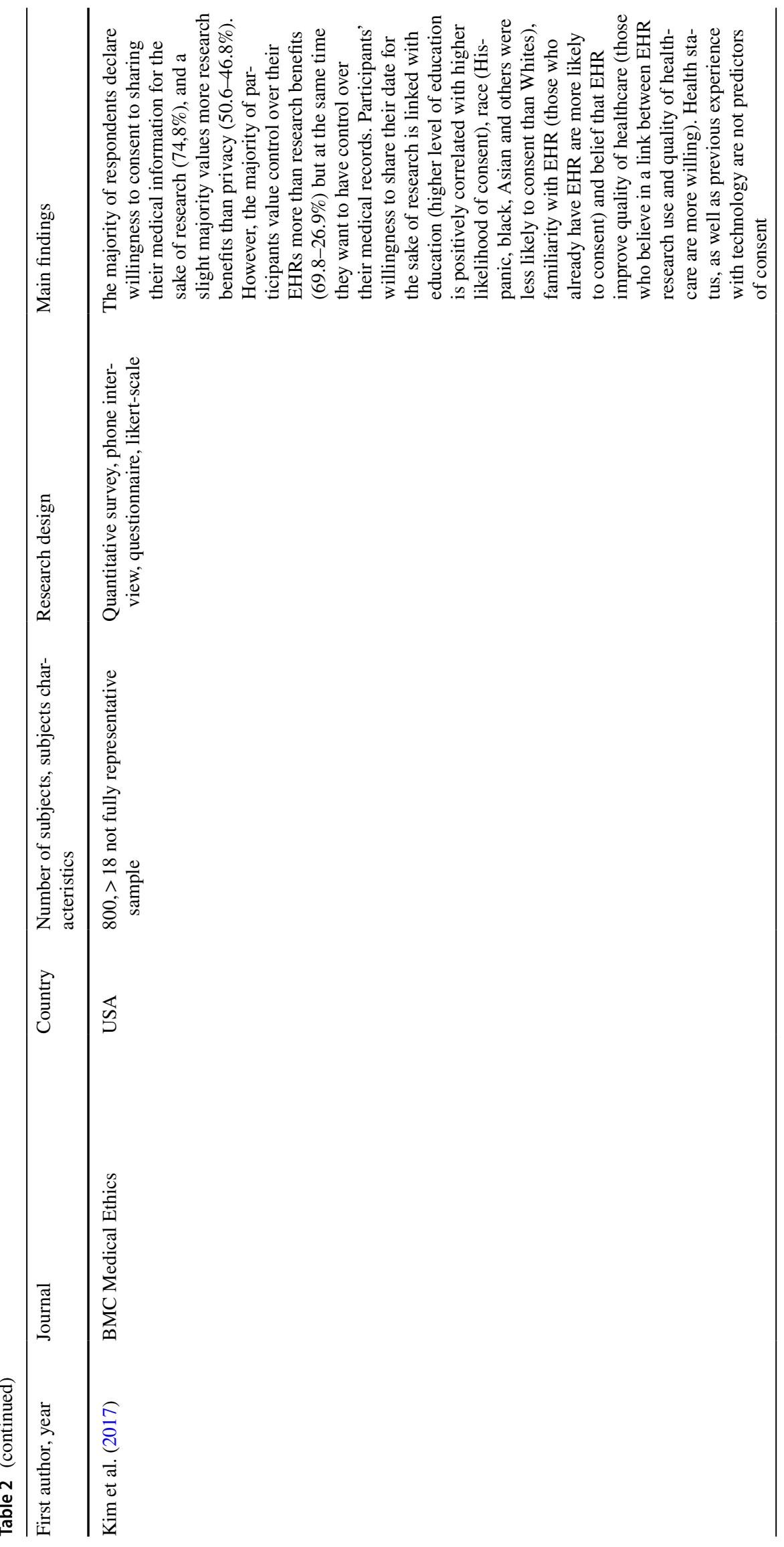




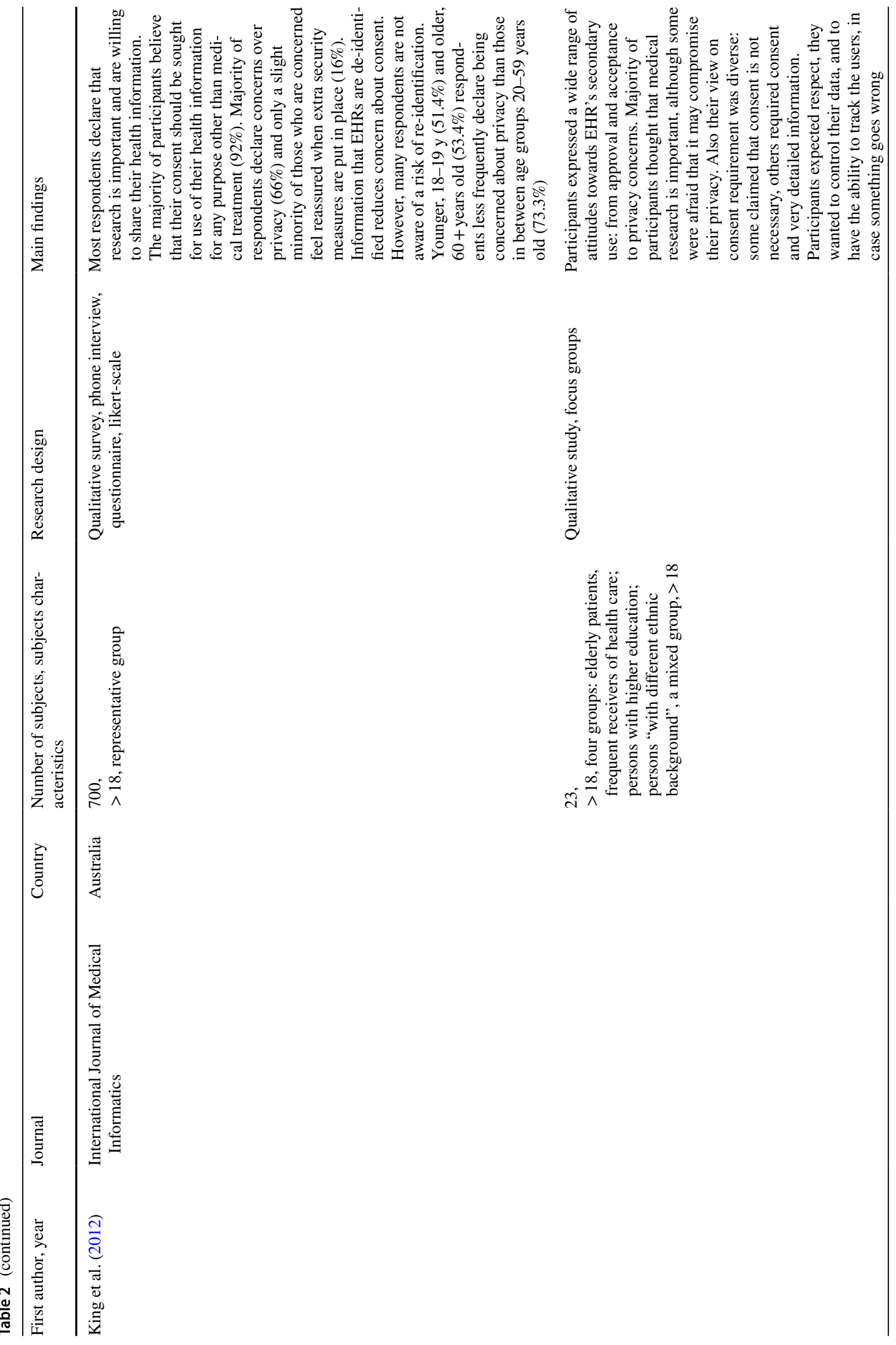




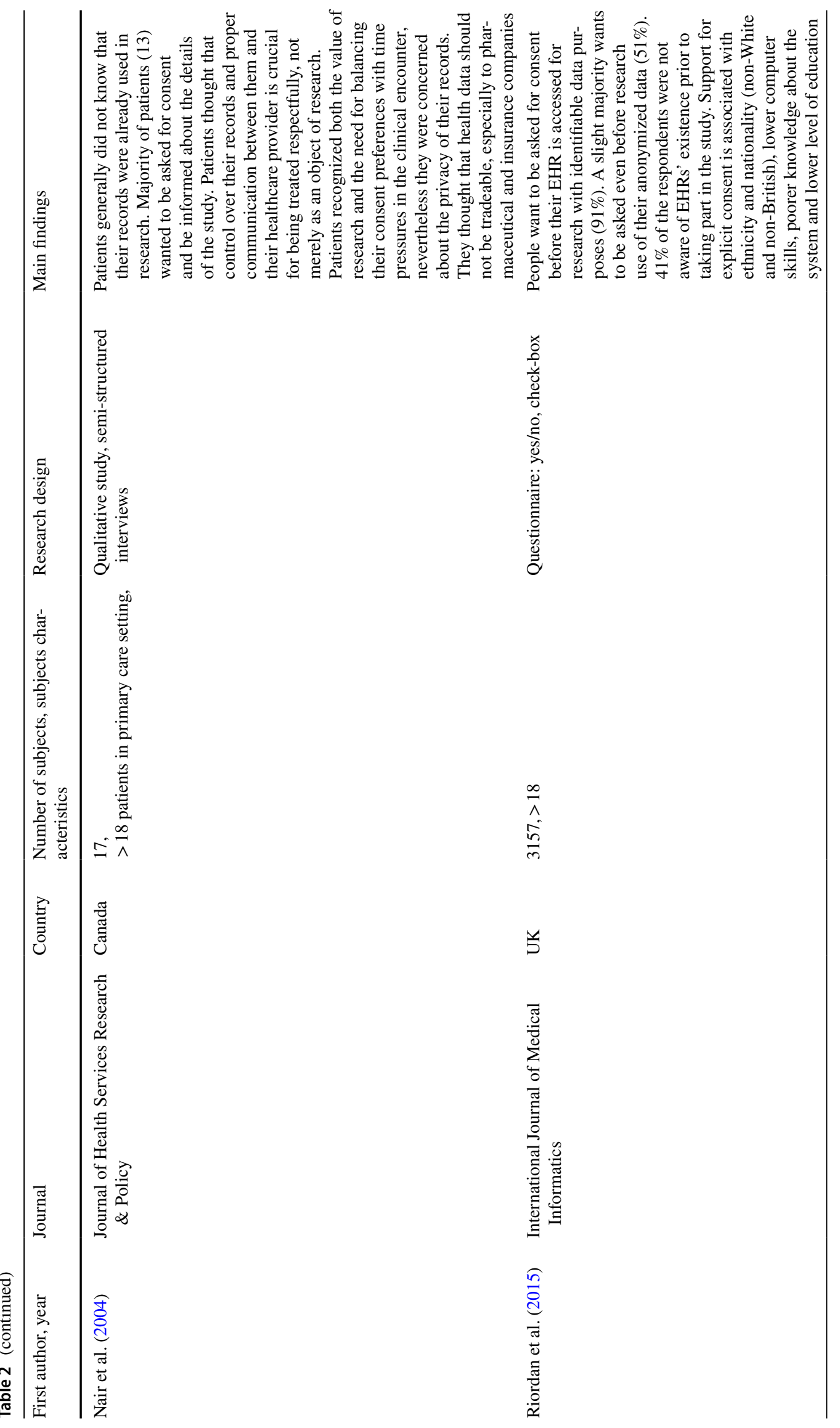




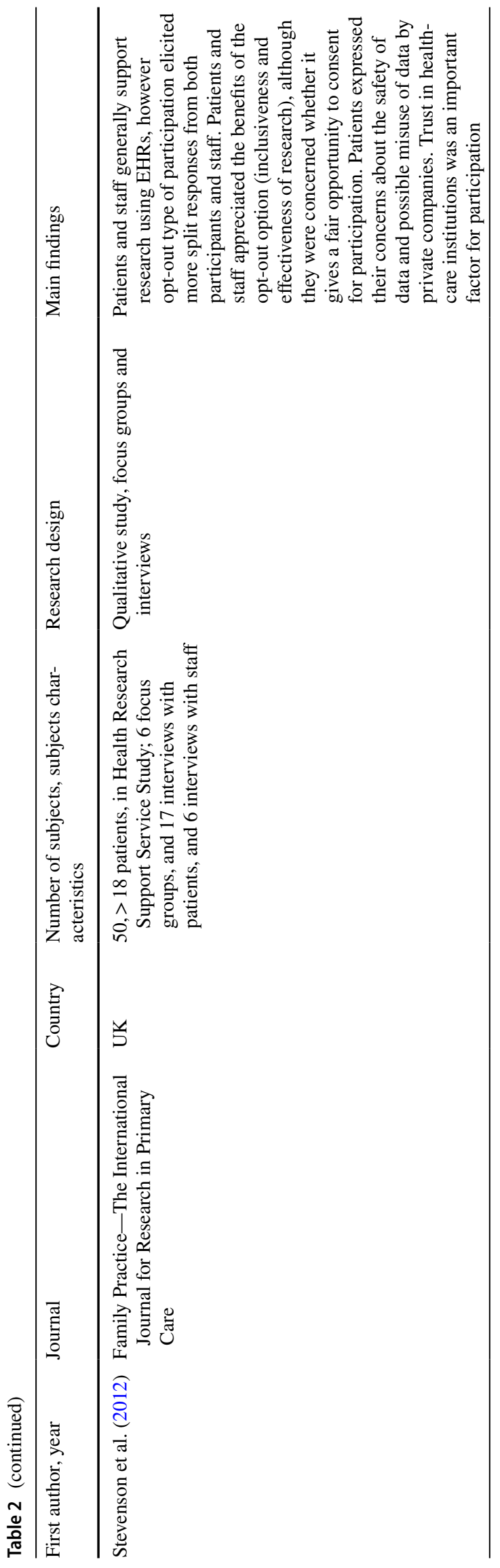

等 Springer 


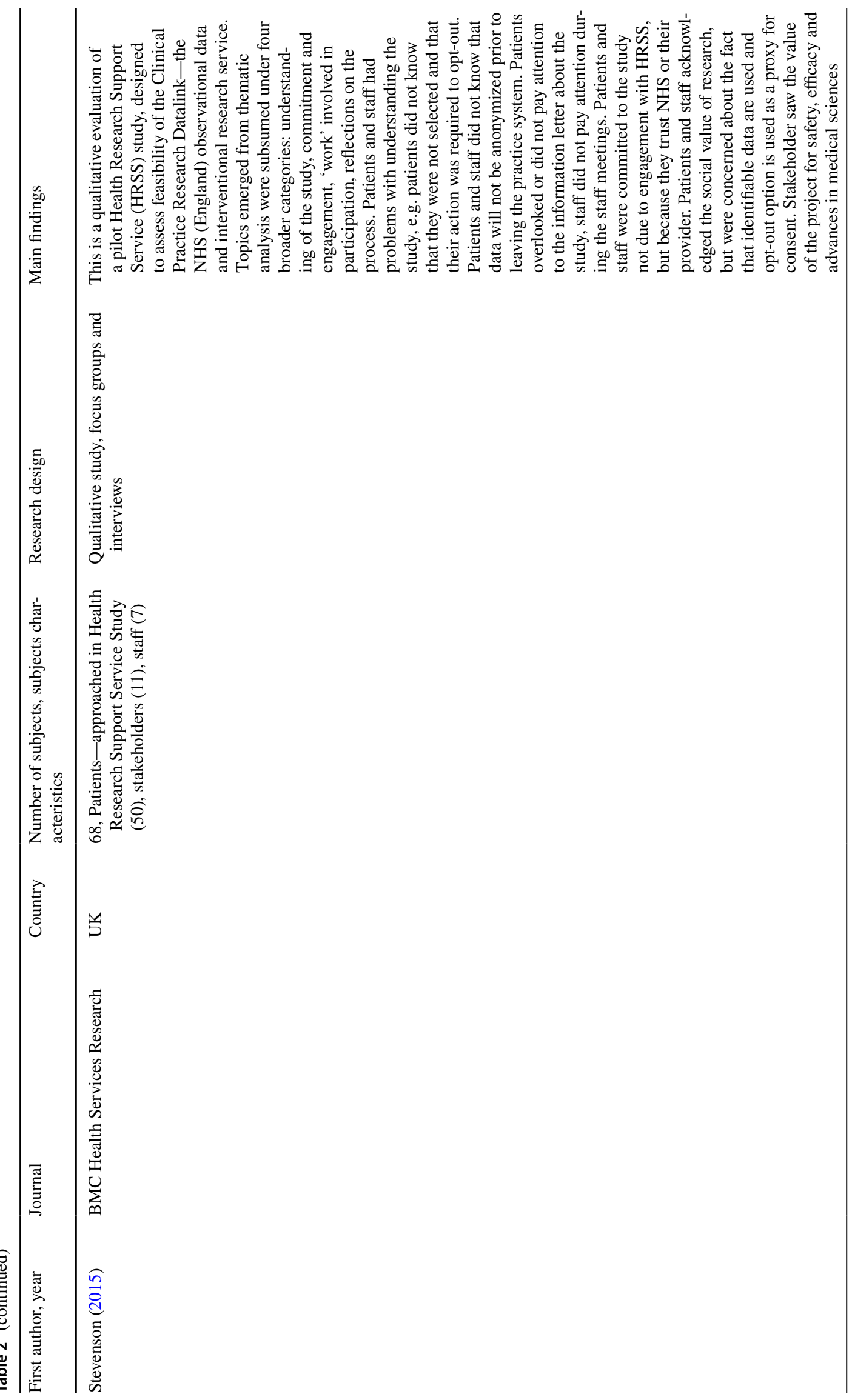




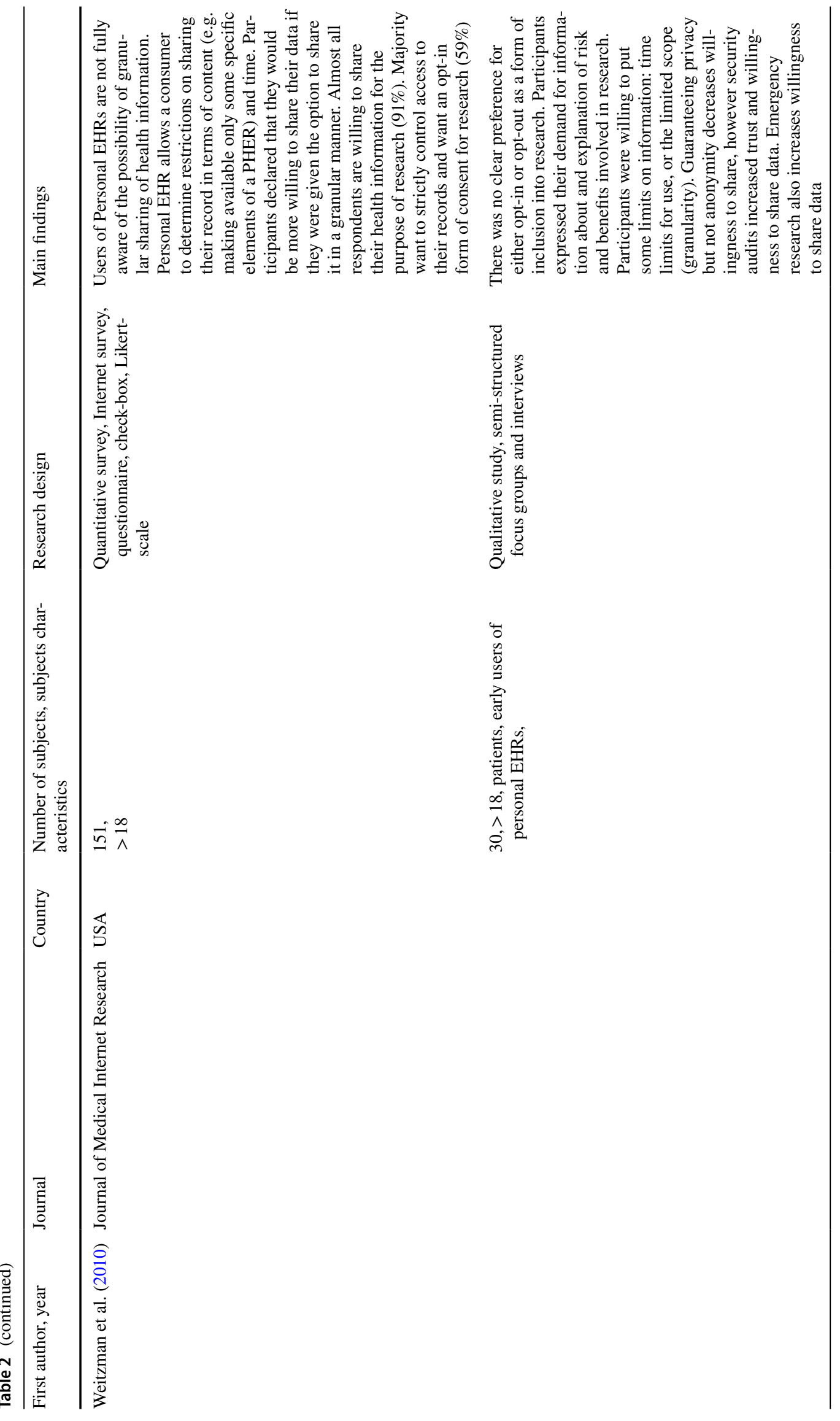




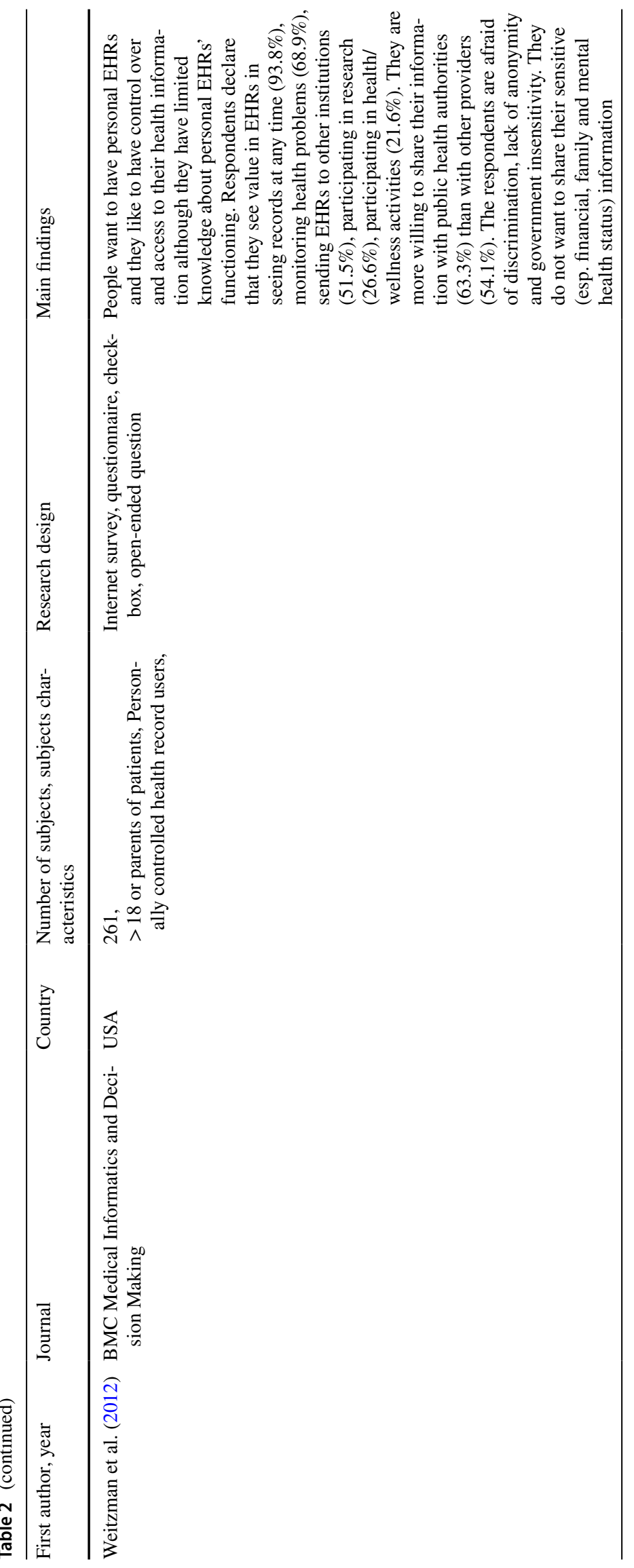






等 Springer 
and legal issues of Big Data, such as the solidarity-based approach (Prainsack and Buyx 2013) and the concept of group-privacy (Taylor et al. 2016). These new approaches seem to be more useful for analyzing the specific issues of data analysis. However, in response it could be pointed out that a qualitative systematic review is better served by a set of general inclusion criteria. This increases sensitivity of a search and is more consistent with the goal of identifying possibly the broadest spectrum of ethical issues.

Replying to the second objection, it can be said that probably there is no ethical framework that is completely culturally neutral and fully universal. Nevertheless, the four-principles framework allows to map the most general ethical tensions present in biomedicine that can occur in clinical settings at every latitude; the tension between: individual interests and interest of society (respect for autonomy versus justice), prospect of benefit and the risk of harm (beneficence versus non-maleficence), individual will and medically defined wellbeing (autonomy versus beneficence). Therefore, this is a useful instrument for detecting the ethical content in a paper, and as mentioned above, we consider the four-principle framework only as a signal for ethical issues. Using the Beauchamp-Childress framework does not imply adopting any specific ethical position. Moreover, our analysis has an inductive character. Therefore, we consider as ethical issues also those issues that were considered ethical by the authors of the analyzed papers, and in our results we go beyond this framework. Furthermore, the BeauchampChildress framework has already been successfully used in other systematic qualitative reviews (Klingler et al. 2017; McLennan et al. 2018; Strech et al. 2013).

Another limitation of our study is that we did not include papers that were not published in English and, therefore, we could have lost some important ethical aspects of EHRs that appear in other cultures, outside the Anglophone world. We agree that the full picture of ethical issues concerning EHRs must be supplemented by cross-cultural studies. However, it should be noted that in the case of non-English publications, there may be uncertainty in the correct interpretation of ethical aspects, precisely because of language or cultural differences.

\section{Results}

\section{Search results}

Searches in Medline Ovid, Embase, and Scopus (22/03/2018) identified, after duplicates had been removed, resulted in 1007 potentially eligible documents (see Fig. 1 presenting the results of all phases). The title-abstract screening resulted in 271 documents for full-text screening ( 2 were unavailable). The final sample of papers meeting the inclusion criteria was 52 documents. They were divided into 3 groups based on the nature of a paper. The first group consists of 37 policy papers that either discuss a certain policy proposal and provide ethical justification for it or discuss general ethical framework for EHRs research and suggest certain policy proposals, or describe how to meet policy and ethics requirements in conducting research using EHRs. The term "policy paper" refers exclusively to the content of a text; a policy paper could be a commentary, an original study, or a book chapter. The remaining 15 empirical papers are a sample of 9 papers containing qualitative data analysis and a sample of 8 quantitative data summaries, which means that 2 papers presented mixed qualitative and quantitative research. We applied the CCM to devise a grid of categories (Table 1) based on all 52 papers. In addition, we provide a narrative summary of 15 empirical papers (Table 2).

\section{Qualitative analysis results}

In the qualitative synthesis, we distinguished 74 specific categories that cluster around 11 general topics (main categories are marked by capital letters A-K, specific categories are marked by a capital letter and a number: A1-K74, see Table 1, and Supplement 1. The Full Grid-this last document allows to estimate the frequency of each category in all the papers and saturation of individual papers with ethical issues. One interested in a particular issue can also locate relevant papers via list of references in the Supplement 1). Below we describe both main categories and subcategories.

\section{Rationale for research using EHRs (A1-A4)}

The first distinguished category is related to the intrinsic and instrumental values of EHR-based research that are mentioned in the included papers. The first reason for implementing EHR-based research is public interest (A1). EHRbased research is deemed instrumental in improvement of public health as well as in increasing efficacy and efficiency of healthcare. The second reason is efficiency and efficacy of EHRs research (A2): EHR-based research is relatively inexpensive and this kind of research can circumvent shortcomings of randomized clinical trials by providing researchers with data about all groups of patients and in that way contributing to generalizable knowledge. The included papers also discuss the fact that research with EHRs can give us a comprehensive picture of a healthcare system, and therefore allow for a more equitable allocation of resources (A3). Finally, the included papers discussed the fact that EHR-based research also promises benefits to the private sector: private hospitals, as well as insurance and technological companies (A4). 
Fig. 1 PRISMA flow diagram

\section{Identification}

MEDLINE OVID

$22 / 03 / 2018$

584
Scopus

$22 / 03 / 2018$

538

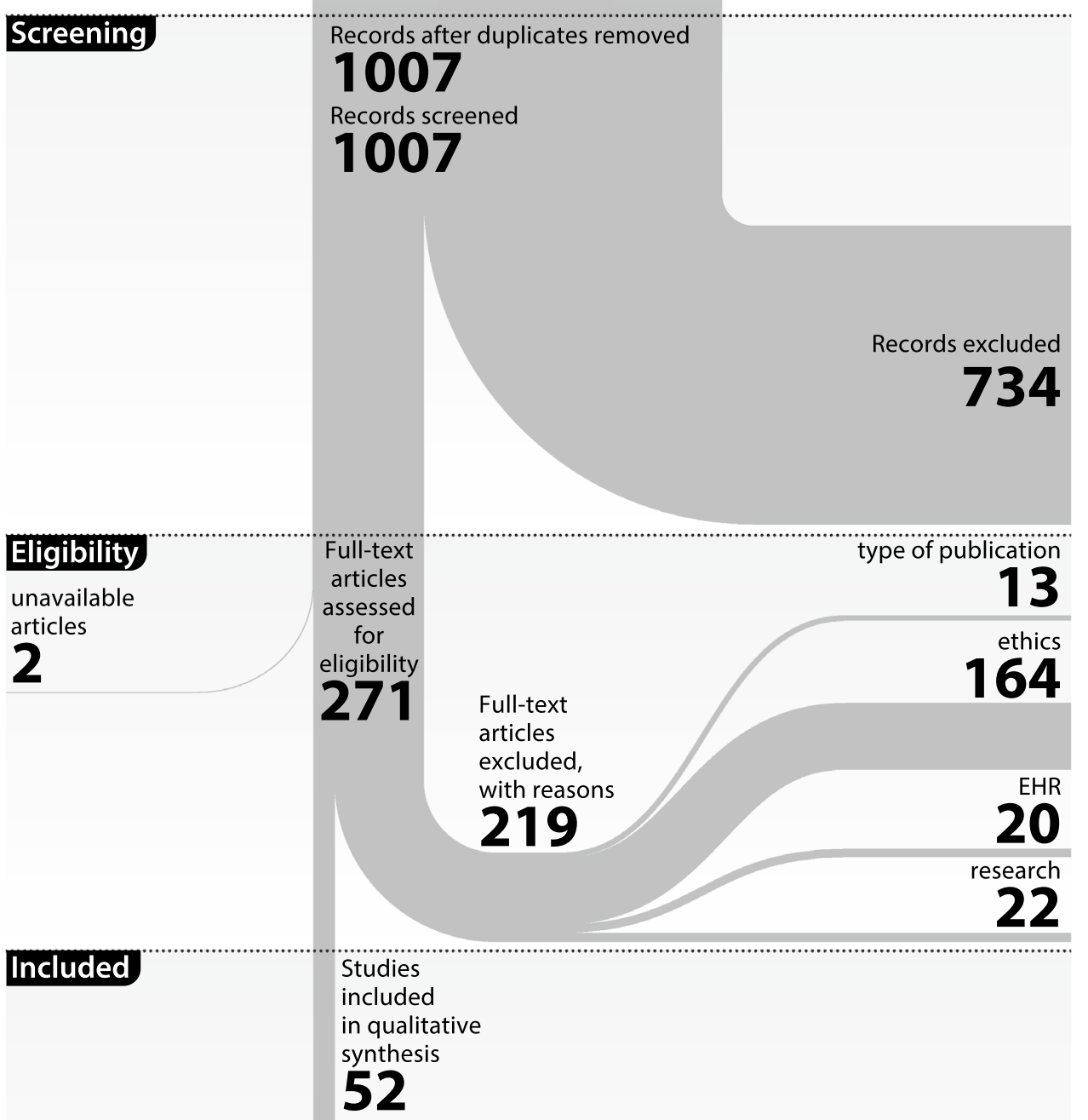

Empirical papers: narrative summary

\section{Factors affecting research use of EHRs (B5-B10)}

Even though EHR-based research is valuable, its implementation is not always easy. The papers we analyzed discussed various factors that can either facilitate this kind of research or obstruct it. The most frequent factor that impedes EHRbased research is regulations that put a burden of administrative work on researchers (B5). This could be especially 
cumbersome in multicenter international research, where there are unharmonized regulations and multiple research ethics committees. Moreover, a research project can fall within the blurred lines of the distinction between research and quality improvement, making it even more difficult for researchers and research ethics committees to decide which regulatory framework should be used.

Policy makers, however, are well aware that regulations can put too heavy a burden on researchers' shoulders, and the included papers also discuss regulatory facilitations and instrumental support of EHR-based research (B6). The support spans from administrative instruments such as abbreviated ethics review and waivers of informed consent requirement to financial investments into technological infrastructure and political support for embedded research. Financial and infrastructure investments are necessary because EHR-based research could encounter technical difficulties with implementation with regards to amount and versatile character of data, data quality, as well as other organizational problems (B7). A separate issue that we distinguished in the included papers is factors that hinder informing participants and obtaining their informed consent. These factors are related to ineffective outreach to participants, insufficient communication with vulnerable and underprivileged groups, problems with processing and documenting consent, especially in regards to tailoring consent to individual preferences (B8).

There are also two context-dependent factors of implementation of embedded research: public awareness (B9) and researchers' attitude (B10). The public can favor EHR-based research or oppose it. Public awareness is also associated with previous experience with EHRs and healthcare, with information policies and practices, as well as multiple individual factors such as confidence in one's computer skills, perception of sensitivity of health data and level of risk. Also, researchers' attitudes (B10) is also a factor that could influence the conduct of EHR-based research. Researchers recognize ethical and legal challenges of embedded research and designing research protocols and they try to balance the imperatives to benefit society and to develop science. However, sometimes they can come to a conclusion that administrative and regulatory barriers make their research projects unworthy of pursuit.

\section{Data management (C11-C18)}

We found several aspects of ethical data management in the literature. First of all, the included papers discuss an issue of safe and secure data storage. There is a variety of specific procedural and technical security measures (C11) such as firewalls, data safe havens and secure data access that can be deployed. Their main goal is to control access to data and protect patients' confidentiality while at the same time streamlining legitimate research. Data used in research has different levels of identifiability (pseudonymized, de-identified, minimized data sets, aggregated data sets) at different stages of research (C12). Some elements of EHRs can contain sensitive information about a patient (C13), such as information about mental illness, fertility, face images, free text with references to third person. Also, quality, quantity and integrity of data (C14) has ethical importance, since researchers can draw conclusions that are meaningful and beneficial to wide social groups only when the data are representative and of good quality. Poor data quality can be also considered as a waste of resources. A separate problem that we discerned in the literature, is data ownership, management and curation (C15). This category encompasses an issue of data control and maintenance, and the possibility of selling data. Meaningful sharing of data (C16) means that data is a valuable resource that can be wasted, when it is not properly used, or when it is collected in such a data format that prevents sharing. We also distinguished in the literature a separate issue concerning data extraction and transfer (C17). From an ethical point of view it is important who has access to health data before it is extracted for the purpose of research, which elements of an EHRs are extracted. If the data contain sensitive information, who is responsible for storage and extraction of data: healthcare workers, researchers or any third entities, as well as who is responsible for decisions about extracting or using data of particular people or groups. This problem is linked to legitimacy of uses and users (C18). Medical professionals, researchers, private companies, healthcare providers, data institutes, disease foundations, governments and patients themselves can use EHR for various legitimate and (potentially) illegitimate purposes, such as research and development, marketing, and education. Empirical research shows (Table 2) that patients and participants sometimes insist on higher ethical scrutiny for certain users and uses. For instance, research use of EHR data by private companies can be considered less legitimate than use by university researchers.

\section{Impact of digitalization on healthcare system providers' operations and patients' engagement (D19-D24)}

Digitalization of healthcare systems and implementation of embedded research impacts healthcare providers and alters their relationship with patients. This impact of digitalization has also an ethical dimension that is discussed in the literature. First of all, EHR-based research changes professional relationships within institutions and between institutions (D19) in a way that blurs the distinction between practice, research and public health activities. Moreover, healthcare workers have to face new responsibilities and adapt to new practices in regard to data processing. As reported in the literature, digitization of health records and 
embedded research poses yet another challenge to communication with patients (D20), who have to be informed about data policies, including policies on data storage, sharing, and roles of a physician and provider as intermediaries between researchers. As is indicated in the literature, this process must foster trust between healthcare professionals and patients. Trust is crucial because patients should be still willing to share with their physicians all sensitive information that is important for their health. Another category that we distinguished in the included papers is directly connected with already discussed alterations in healthcare practice: digitization of health records brings new ethical responsibilities of the medical staff (D21). Healthcare professionals have to understand ethical challenges associated with EHRs and embedded research, especially if they are involved in data processing. Involvement into data processing, notably, when it directly concerns cooperation with researchers, can also be a source of moral distress and unwillingness to share patients' data outside the context of their current care. Moreover, as reported in the analyzed papers, embedded research also requires additional work from staff and patients (D 22). Staff is involved in data curation and informing patients; patients have to respond to research invitations and fill out additional documents. In both cases, all these activities are discrete forms of resource allocation. Another aspect of embedded research, discussed in the literature, is the pivotal role of patients (D23). LHSs are sustainable in the long run only when there is broad patient participation, public acceptance and support. Some included papers addressing this issue underline the necessity of patient involvement into policy-making and implementation. Finally, a few papers included in our sample envision and discuss an idea that we called "digital patient-citizenship" (D24). Digital patientcitizenship is a proposal to encourage patient participation in research activities, research oversight, policy making and all the responsibilities associated with curation of data. In that vision, a personal EHR is a tool of patient empowerment. It is also an attempt to take a bit broader look at EHRs, not merely as an element of the healthcare system, but an element of contemporary digital culture.

\section{Risk, harms and burdens of research with EHRs (E25-39)}

Although usually it is believed that embedded research does not pose more than minimal risk, due to the fact that research use of EHRs is not associated with additional risk other than everyday medical practice, we found fifteen different categories of risk posed by EHR-based research. The most obvious seems to be risk to privacy (E25), then risk to patient autonomy (E26), when one loses control over one's data. Because data use can violate a patient's beliefs and values, it is associated with a risk of dignitary harm (E27). Risk for patients encompasses also harmful use of data (E28), e.g., when one's data is used by a misleading pharmaceutics marketer, or when one loses a job after a leak of health information. In the papers we also encountered a risk of legal (E29) and psychological harm (E30). Some authors discuss information risk (E31) that is associated with the fact that patients could be insufficiently informed about research or that they can misunderstand provided information. Information risk category encompasses also a common situation of social science: it is difficult to study patients' opinions and preferences, since people usually form opinions during research itself. Another category of risk that we encountered in the literature is a risk of exploitation (E32), when one's data are used without consent for commercial purposes. In the included papers we also identify undue pressure to participate in research (E33). This kind of risk is closely associated with the risk to the whole healthcare system: when patients realize that their data are being used for illegitimate purposes or by illegitimate users that could undermine trust between the healthcare system and patients (E34) and lead to a massive drop out from research activities. Undermined trust can also result in other risks to patients. For example compromised care (E35) because patients withhold certain information from healthcare professionals; compromised care can be also a result of burdens that are associated with processing information and deflection of attention from a patient. We also distinguished a category of group mediated risk (E36). Research using EHRs provides detailed health related knowledge about individual patients, as well as about certain groups (e.g., ethnic minorities) and populations. Based on this knowledge, individuals and groups can be stigmatized and discriminated against by, for example, refusal of certain services. We also found a risk of financial conflict of interest (E37): researchers can take advantage of access to data and sell it. This kind of risk is more often mentioned by respondents in empirical research. Finally, their EHRbased research also poses risk to healthcare providers. First, because they can face additional work and administrative burden (E38). Second, disclosure of information about providers can sometimes undermine their reputation and commercial interests (E39).

\section{Measures for subject protection (F40-F46)}

The included papers also discuss a variety of protective measures against negative consequences of participation in EHR-based research: independent review by a research ethics committee or privacy board (F40), requirement for informed consent or authorization (F41), legal privacy regulations and ethical guidelines (F42), risk assessment procedures (F43), primary care provider consent (F44), as well as community or patients' panel consent (F45). When one considers a healthcare institution as a participant of embedded research, then some authors also propose providers' consent mechanism (F46) that 
would be devised for protection of healthcare providers' interests, such as control of access to data.

\section{Type of consent (G47-G54)}

The papers that we analyzed discuss several different types of consent in the context of EHR-based research. The first problem associated with research is approaching a patient and initiation of contact (G47). Embedded research might require different levels of patient involvement and in the literature a broad variety of options spanning from no need for consent, through opt-out to fully-informed and document consent (G48) are discussed. A separate issue is documentation of consent for EHR-based research and whether it should be a written document or just a verbal consent that is then marked in a patient's electronic documentation. The included papers discuss also a seemingly opposing approaches to consent: broad consent (G50), where a patient agrees in advance for a whole spectrum of different and unknown research belonging to a certain category, and interactive and granular consent (G51), when a patient can actively select not only studies that she wants to participate in, but also pieces of information from her EHR that she is willing to share with researchers. The papers we analyzed also discuss such issues as when should researchers be able to obtain retrospective consent (D52) or what conditions have to be met to waive this requirement. Finally, papers also mention proxy consent (G53) and assent (G54). The latter two issues, however, are not thoroughly discussed in the literature.

\section{Content of consent (H55-H60)}

As a separate topic we distinguished a category of content of consent $(\mathrm{H})$. This category encompasses all pieces of information that a patient could or should be provided with in EHR-based research. Several of the included papers discuss issues such as the issue of data management, purpose of research, possible future use of data, storage and sharing details (H55). Some authors include additional items to the list of items that should be discussed in a consent form: security measures (H56), benefits, risks and burdens (H57) associated with research, as well as commercial application of data (H58). Only 4 papers discuss the issue of communication of study results (H59), and 2 mention that informed consent should contain information about research funding sources (H60).

\section{Reasons and motives for participation in EHR-based research (162-164)}

Empirical research on patient attitudes towards EHR-based research is summarized in Table 2. Generally, patients or respondents representing the general public express their willingness to make one's EHR available for the sake of research, as well as express other motives, such as altruism and solidarity, support for science, and health of future generations (I61, I62). Decisions to participate in research depend on trust of involved institutions (I63) and personal and sociodemographic factors such as race, education, income, living in a city, and employment status (I64).

\section{Emotions experienced as a result of reflection on EHRs and/ or participation in HER-based research (J65-J67)}

Mainly empirical papers allowed us to distinguish also the emotional component of attitude toward EHRs and EHRbased research. Some patients and participants express their positive emotional attitude towards research encompassing a sense of comfort, trust, and even excitement with new vistas for biomedical research (J65). Others responded with emotions indicating negative attitudes toward research, such as discomfort, wariness, lack of commitment, anxiety and confusion (J66). Finally, there were also patients and participants whose attitude was inconsistent and they expressed enthusiastic support for research and great concern for privacy, felt uncomfortable about the fact that facilitation of research decreases the level of personal control over data (J67).

\section{Ethical values, rights, and obligations (K68-K74)}

Finally, we identified 7 explicit ethical topics discussed in the context of EHR-based research. 49 papers discuss the traditional patient rights to information and to autonomous choice, for instance to donate one's EHR to be used after the donor's death (K68). Next most frequently discussed issue is information, public education and public engagement (K69) that is also associated with such values as transparency, empowering of patients and communities. In the context of embedded research, the ethical principle of beneficence (K70) is also discussed. In addition, as some authors suggest, not only researchers and healthcare workers are obliged to conduct research for the public benefit, but patients are also under similar obligation to participate in low risk embedded research. Another quite frequently invoked ethical principle is the principle of justice (K71), which is translated in the context of EHR-based research into fair benefit sharing, fair recruitment and protection of vulnerable groups and individuals, and involving local communities in the process of research. A few papers discuss the problem of research integrity and respect for intellectual property (K73). Less frequently, a right to optimal healthcare and right to clinical judgment is discussed, as well as a right to compensation (K74). 


\section{Discussion}

\section{Ethical challenges of EHR-based research}

The grid of categories cannot substitute normative discussion on how EHRs should be used in the course of research. Moreover, our grid of ethically relevant issues does not by itself inform which issues are more, and which are less, important, and how to solve a possible conflict between several ethical values. Therefore, the results of our study should be considered as a point of departure for a normative deliberation, not the conclusion or solution for problems. The main merit of our study is the presentation of how accessibility of the EHR presents itself in everyday research and health care practice, and what elements of this complicated picture bear ethical significance.

Two main questions emerge from the grid of categories. The first one considers tension between individual and societal interests in the context of population research. On the one hand, we see a trend to empower individuals in their decision making, through new digital technologies (G61). On the other hand, policy makers and researchers aim at streamlining research and relaxing obstructive regulations, seeking quick delivery of generalizable knowledge (B6). This tension between individual rights and public health interests seems to be even more poignant in the time of public health emergencies, such as COVID-19 pandemic. In our opinion, this tension can be overcome, perhaps, by the idea of digital citizenship and recognition of patient contributions to the LHS (D23-24). The idea of digital citizenship appears only in 14 papers that we analyzed. However, the concept of citizenship is a promise to reconcile autonomy of an individual citizen, whose unalienable rights should always be respected by the state, and the fact that a citizen is always a member of the political community. Citizen participation in community life aims at defining and realizing the common good. Yet, it is still a promising idea and is not clear how exactly it can be implemented into policies, regulation and practice.

The second question is: does implementation of LHSs fueled by EHRs simply exacerbate already existing ethical problems and what kind of new challenges for policymakers, healthcare providers and researchers does it create? In the following discussion, we attempt to provide some additional context for these two issues and shed some light on a possible ethical justification for selected ethical categories from the grid.

\section{Towards digital citizenship?}

\section{Granular approach to consent}

Legal regulations in many countries (e.g. US and EU) already allow for modification or even a complete waiver of informed consent in the case of EHR-based research. Nevertheless, a waiver of informed consent disables individual control over data and can undermine public trust in research and healthcare institutions. The idea of empowering patients by giving them access to their data through IT tools is in line with the empirical studies that show patients want to know what is happening with their EHRs and that information policies play an important role in preserving trust towards healthcare institutions (Tale 2). Technology opens an opportunity to a more granular approach to informed consent (G51). It means that a patient, logging through a patient interface, is able to choose which elements of her records can be accessed by researchers and for how long. Such digital tools are already applied in research and clinical practice (Shelton 2011, Wallace and Miola 2021). This is in congruence with Neal Dickert's and his colleagues analysis that distinguishes seven different functions of informed consent. Informed consent 1 . Makes the process of research transparent; 2 . Allows to control and authorize research; 3 . Gives a patient opportunity to participate only in those research projects which conform to her values; 4 . Protects and promotes welfare; 5. Promotes public trust, 6. Is required by regulations, and researchers who follow regulations are protected, and 7. Promotes research integrity (Dickert et al. 2017). Technological advances therefore give us an opportunity to balance individual control and public responsibility, because it seems reasonable to think that not all functions of informed consent have to be performed in all circumstances. There are some tradeoffs that a society or community could negotiate. For instance, one can agree that public health goals might require obligatory accessibility of health information concerning infectious diseases, especially in the time of pandemic, but other elements of an EHR could be under patient's control.

\section{Exercising control over one's data}

The empirical studies that we analyzed also reveal that some patients hold a belief that data ownership is an appropriate instrument to control their data (Table 2). The idea of health data ownership has been discussed thoroughly, especially in the US context (Evans 2011; Haislmaier 2006; Hall and Schulman 2009; Hall 2010; Kish and Topol 2015; Mirchev et al. 2020; Purtova 2015, 2017; Rodwin 2009). It was argued that every EHR as a byproduct of therapeutic encounters belongs to a patient and medical facility (Haislmaier 2006; Hall and Schulman 2009; Hall 2010). An independent intermediary-EHR bank-can manage collection, exchange and access to databases for researchers, and other parties. The revenue would then be shared among the patient, the medical facility, and the EHR bank. Thus, it was thought, an invisible hand of the market will give a spur to the economy and research enterprise at once. 
Nonetheless, this idea has been criticized for several reasons. The proponents of public interests argued that privatization of EHRs would increase the cost of public health and epidemiological research, as well as result in biased, not representative, research samples (Rodwin 2009; Evans 2011). Barbara Evans argues that in the US legal context property right is not an absolute one, and in reality it does not give an individual stronger privacy protection instruments (Evans 2011).

Generally, two different regimes of data protection with some variations can be distinguished (Painsack 2019). In the first regime an individual has control over data. This control can be exercised by property rights (the US) or data can be considered, as in the EU, an inalienable individual possession protected by civil rights (Painsack 2019). The second regime of data protection introduces an element of collective control over data, and as Prainsack argues, this element of collective control can be reconciled with the concept of data as inalienable possession. A similar idea was also discussed in one article included in our sample (Grande et al. 2014). Public data stewardship is an element of digital citizenship, where the community as a whole can balance individual rights and common good through deliberation and decisions can be made in a legitimate political process. Thus the problem of who can use people's data and how is left neither to paternalistic protection of public health nor to purely economic forces. Data stewardship, nonetheless, requires additional education efforts, and can be probably implemented in societies with a high level of public trust and solidarity (K70-71). It seems that as societies, we need digital education that would explain the benefits and risks of new technologies.

\section{Healthcare provider as a research participant}

The problem of data stewardship is even more complicated, because a community has to balance not only individual and societal interests, but also interests of corporate entities. Gregory Simon realizes that a healthcare provider cannot be unconditionally included into embedded research, because this kind of research also entails some risks for the whole institution (Simon et al. 2017, Piasecki \& Dranseika 2021). We discussed the problem thoroughly in a separate publication, and we proposed three different strategies of finding a balance between a provider's professional obligation to contribute to the development of healthcare and duty to protect important interests of the institution. The first approach is the self-regulating model, probably the most suitable for free market driven healthcare systems, like in the US, where balancing is managed by healthcare providers themselves. The centralized model is more suitable for a centralized public healthcare system. In this model, the process of managing patients' data is governed by a state body. The most democratic and patient- empowering approach is possible in the mediating model, where providers, the state and the citizens can negotiate what kind of data can be made accessible to researchers (Piasecki \& Dranseika 2021).

\section{New ethical challenges for policy makers}

\section{Research disrupts practice}

EHRs are a key element of collecting data in a systematic manner during medical practice and then using these data to develop generalizable knowledge. However, in everyday life researchers usually do not have direct access to EHRs and practitioners' access to EHRs is strictly regulated, as well. Thus, the use of EHRs presents a set of practical and ethical challenges: who can access an actual patient's record and to what extent? How are the data from the record extracted and stored? (Evans 2011) How does this process of data extraction for the purpose of research influence everyday healthcare provider operations? (D19-22) How to contact patients and how to provide them with information about the research projects? Moreover, the empirical research with healthcare staff that is involved in processing and sharing the data reveals that healthcare professionals are not comfortable with sharing data outside the context of healthcare (Stevenson 2015) (D21). They are trained to keep patients' data confidential. They build their trust relationship with patients on the basis of this commitment to professional confidentiality. As it has already been mentioned, building an LHS requires, then, not only technological tweaks and contact with patients, but also changing the organizational culture of healthcare providers (Foley and Fairmichael 2015).

In a LHS healthcare professionals: physicians, nurses, administrative clerks are assigned new roles (F29, K69-72). They are becoming a part of the LHS, obliged to provide additional information to researchers, overview and assess collected data and handle contacts with researchers. These new assignments transform ethical responsibilities of healthcare workers. The healthcare professionals have to accept and internalize these new responsibilities in order to make the LHS run in an efficient and ethical way. Similarly, patients have to recognize that quality of care, effectiveness and safety of provided therapies depend on their contribution and cooperation.

\section{Ethical framework for the LHS}

But it seems that these relatively miniscule changes in the healthcare systems constitute a more general ethical problem. Namely, what is the role of healthcare and healthcare systems in modern societies? Is healthcare a human right and a response to individual health needs? Is healthcare just one of the different goods that are available in the 
free market economy? (Daniels 2001) This question is not always dealt with directly. It seems that when one discusses a new ethical framework for LHSs, this implicitly answers it (Faden et al. 2013a; b). A raw fact of altered accessibility of EHR, accumulation of data and rising computing powers change our approach to clinical ethics, research ethics and public health ethics (Piasecki \& Dranseika 2019a). We are facing a stark choice: what values our regulatory and technological environment should espouse: give precedence to individual interests or promote the public good? Currently, in both clinical and research ethics, the binding principle is still that of precedence of the individual interest. The new approach seems to take a different turn and underscores the moral importance of public good. In public health ethics the main goal is good of the community as a whole. But in the pursuit of that public good we cannot entirely discard the value of individuals (Kass 2001, Piasecki \& Dranseika 2019b). And this new question "What should be the organizing principle of EHRs use in LHS?" also emerges from the results of our study. This issue has its consequences on all levels of the healthcare system and it affects not only the framing regulations, but also professional roles of healthcare workers, and patients' attitudes and expectations.

\section{Conclusion}

In this systematic review, we have presented a wide spectrum of ethical issues involved in EHR-based research. All these problems are related to the main issue: how to manage access to health information. The reviewed literature allowed us to capture different aspects of access management and perspectives of different stakeholders. In conclusion, it can be said that most of the problems arise from a rapid cultural change. The framing concepts of privacy, as well as individual and public dimensions of beneficence, are changing. We are currently living in the middle of this transition period. Human emotions and mental habits, as well as laws, are lagging behind technological developments. In the medical tradition, individual patient's health has always been in the center. Transformation of healthcare care, its digitalization, seems to have some impacts on our perspective on health care ethics, research ethics and public health ethics.

Supplementary Information The online version contains supplementary material available at https://doi.org/10.1007/s11019-021-10031-6.

Acknowledgements Authors thank Patrycja Brzukala and Ewelina Sadowska for their contribution to the tile-and-abstract phase of the review. We thank Phyllis Zych Budka for linguistic edits.

Author contributions All authors had their contribution into conception of the study, collecting of data, reviewing the manuscript and approved the final version. JP prepared the manuscript. All other details of authors' contribution is described in the paper.

Funding This systematic review is a part of the project funded by the National Science Centre, Poland, 2015/19/D/HS1/00991.

Data availability All essential data are available in supplementary materials.

\section{Declarations}

Conflict of interest The authors declare no conflict of internets.

Open Access This article is licensed under a Creative Commons Attribution 4.0 International License, which permits use, sharing, adaptation, distribution and reproduction in any medium or format, as long as you give appropriate credit to the original author(s) and the source, provide a link to the Creative Commons licence, and indicate if changes were made. The images or other third party material in this article are included in the article's Creative Commons licence, unless indicated otherwise in a credit line to the material. If material is not included in the article's Creative Commons licence and your intended use is not permitted by statutory regulation or exceeds the permitted use, you will need to obtain permission directly from the copyright holder. To view a copy of this licence, visit http://creativecommons.org/licenses/by/4.0/.

\section{References}

Aicardi, C., L. Del Savio, E.S. Dove, F. Lucivero, N. Tempini, and B. Prainsack. 2016. Emerging ethical issues regarding digital health data. On the World Medical Association Draft Declaration on Ethical Considerations Regarding Health Databases and Biobanks. Croatian Medical Journal 57 (2): 207-213.

Aitken, M., J. de St Jorre, C. Pagliari, R. Jepson, and S. CunninghamBurley. 2016. Public responses to the sharing and linkage of health data for research purposes: A systematic review and thematic synthesis of qualitative studies. BMC Medical Ethics 17 (1): 73.

Anderson, R. 2015. The collection, linking and use of data in biomedical research and health care: ethical issues. https://doi.org/ 10.17863/CAM.31760

Beauchamp, T.L., and J.F. Childress. 2013. Principles of Biomedical Ethics, 7th ed., 480. Oxford: Oxford University Press.

Boeije, H.A. 2002. Purposeful approach to the constant comparative method in the analysis of qualitative interviews. Quality \& Quantity 36 (4): 391-409.

Botkin, J.R., E. Rothwell, R. Anderson, L.A. Stark, and J. Mitchell. 2014. Public attitudes regarding the use of electronic health information and residual clinical tissues for research. Journal of Community Genetics 5 (3): 205-213.

Chassang, G. 2017. The impact of the EU general data protection regulation on scientific research. E-Cancer Medical Science 11: 709.

Clerkin, P., B.S. Buckley, A.W. Murphy, and A.E. MacFarlane. 2012. Patients' views about the use of their personal information from general practice medical records in health research: A qualitative study in Ireland. Family Practice 30 (1): 105-112.

Cohen, I.G., and M.M. Mello. 2018. HIPAA and protecting health information in the 21st Century. JAMA 320 (3): 231-232.

Daniels, N. 2001. Justice, health and healthcare. The American Journal of Bioethics 1 (2): 2-16. 
Dickert, N.W., N. Eyal, S.F. Goldkind, C. Grady, S. Joffe, B. Lo, et al. 2017. Reframing consent for clinical research: A function-based approach. The American Journal of Bioethics 17 (12): 3-11.

Dye, J., I. Schatz, B. Rosenberg, and S. Coleman. 2000. Constant comparison method: A kaleidoscope of data. The Qualitative Report $4(1 / 2): 1$.

El Emam, K., E. Jonker, L. Arbuckle, and B. Malin. 2011. A systematic review of re-identification attacks on health data. PLOS ONE 6 (12): e28071.

El Emam, K., S. Rodgers, and B. Malin. 2015. Anonymizing and sharing individual patient's data. BMJ 350: h1139.

Evans, B.J. 2011. Much ado about data ownership. Harvard Journal of Law \& Technology 25 (1): 69-130.

Evans, R.S. 2016. Electronic health records: Then, now, and in the future. Yearbook of Medical Informatics 1: S48-61.

Faden, R.R., N.E. Kass, S.N. Goodman, P. Pronovost, S. Tunis, and T.L. Beauchamp. 2013a. An ethics framework for a learning health care system: A departure from traditional research ethics and clinical ethics. Hastings Center Report 43 (s1): S16-27.

Faden, R.R., N. Kass, D. Whicher, W. Stewart, and S. Tunis. 2013b. Ethics and informed consent for comparative effectiveness research with prospective electronic clinical data. Medical Care 1: S53-S57.

Foley, T., and F. Fairmichael. 2015. The Potential of Learning Healthcare Systems. Newcastle: Learning Healthcare Project.

Friedman, C.P., Macy, J. 2014. Toward Complete \& Sustainable Learning Systems 2014. https://medicine.umich.edu/sites/default/files/ 2014_12_08-Friedman-IOM\%20LHS.pdf. Accessed 20 Aug 2020

Geissbuhler, A., Safran, C., Buchan, I., Bellazzi, R., Labkoff, S., Eilenberg, K., Leese, A., Richardson, C., Mantas, J., Murray, P., and G. De Moor. 2013 Trustworthy reuse of health data: a transnational perspective. International journal of medical informatics 82(1): $1-9$

Gibbs, G. 2009. Analysing Qualitative. Data Qualitative Research Kit, 176. London: SAGE.

Grande, D., N. Mitra, A. Shah, F. Wan, and D.A. Asch. 2013. Public preferences about secondary uses of electronic health information. JAMA Internal Medicine 173 (19): 1798-1806.

Grande, D., N. Mitra, A. Shah, F. Wan, and D.A. Asch. 2014. The importance of purpose: Moving beyond consent in the societal use of personal health information. Annals of Internal Medicine 161 (12): 855-862.

Haddaway, N.R., A.M. Collins, D. Coughlin, and S. Kirk. 2015. The role of Google Scholar in evidence reviews and its applicability to grey literature searching. PLoS ONE 10 (9): e0138237.

Haislmaier, E.F. 2006. Health care information technology: Getting the policy right. The Heritage Foundation 1131: 1-3.

Hall, K. 2016. UK Gov's hated Care.data project binned. The Register. https://www.theregister.co.uk/2016/07/06/caredata_binned/. Accessed 20 Aug 2020

Hall, M.A., and K.A. Schulman. 2009. Ownership of medical information. JAMA 301 (12): 1282-1284.

Hall, M.A. 2010. Property, privacy, and the pursuit of interconnected electronic medical records. Iowa Law Review 95 (2): 631-663.

Hansson, M.G. 2010. Need for a wider view of autonomy in epidemiological research. BMJ (clinical Research) 340: c2335.

Häyrinen, K., K. Saranto, and P. Nykänen. 2008. Definition, structure, content, use and impacts of electronic health records: A review of the research literature. International Journal of Medical Informatics. 77 (5): 291-304.

Helgesson, G., and S. Eriksson. 2008. Against the principle that the individual shall have priority over science. Journal of Medical Ethics 34 (1): 54-56.

Hill, E.M., E.L. Turner, R.M. Martin, and J.L. Donovan. 2013. "Let's get the best quality research we can": Public awareness and acceptance of consent to use existing data in health research: A systematic review and qualitative study. BMC Medical Research Methodology 13: 72.

Jones, R.D., A.N. Sabolch, E. Aakhus, R.A. Spence, A.R. Bradbury, and R. Jagsi. 2017. Patient perspectives on the ethical implementation of a rapid learning system for oncology care. Journal of Oncology Practice 13 (3): e163-e175.

Kass, N.E. 2001. An ethics framework for public health. American Journal of Public Health 91 (11): 1776-1782.

Kim, K.K., P. Sankar, M.D. Wilson, and S.C. Haynes. 2017. Factors affecting willingness to share electronic health data among California consumers. BMC Medical Ethics 18 (1): 25.

King, T., L. Brankovic, and P. Gillard. 2012. Perspectives of Australian adults about protecting the privacy of their health information in statistical databases. International Journal of Medical Informatics 81 (4): 279-289.

Kish, L.J., and E.J. Topol. 2015. Unpatients-why patients should own their medical data. Nature Biotechnology 33 (9): 921-924. https:// doi.org/10.1038/nbt.3340.

Klingler, C., D.S. Silva, C. Schuermann, A.A. Reis, A. Saxena, and D. Strech. 2017. Ethical issues in public health surveillance: A systematic qualitative review. BMC Public Health 17 (1): 295.

McLennan, S., H. Kahrass, S. Wieschowski, D. Strech, and H. Langhof. 2018. The spectrum of ethical issues in a Learning Health Care System: A systematic qualitative review. International Journal for Quality in Health Care 30 (3): 161-168.

Mirchev, M., I. Mircheva, and A. Kerekovska. 2020. The academic viewpoint on patient data ownership in the context of big data: Scoping review. Journal of Medical Internet Research 22 (8): e22214.

Nair, K., D. Willison, A. Holbrook, and K. Keshavjee. 2004. Patients' consent preferences regarding the use of their health information for research purposes: A qualitative study. Journal of Health Services Research \& Policy 9 (1): 22-27.

Painsack, B. 2019. Logged out: Ownership, exclusion and public value in the digital data and information commons. Big Data \& Society 6 (1): $1-15$.

Piasecki, J., Waligora, M., and V. Dranseika. 2018. Google search as an additional source in systematic reviews. Science and Engineering Ethics 24: 809-810.

Piasecki, J., and V. Dranseika. 2019a. Research versus practice: The dilemmas of research ethics in the era of learning health-care systems. Bioethics 33(5): 617-624.

Piasecki, J., and V. Dranseika. 2019b. Learning to regulate learning healthcare systems. Cambridge Quarterly of Healthcare Ethics 28(2): 369-377.

Piasecki, J., and V. Dranseika. 2021. Balancing professional obligations and risks to providers in learning healthcare systems. Journal of medical ethics 47(6): 413-416.

Pilkington, E. 2019. Google's secret cache of medical data includes names and full details of millions-whistleblower. The Guardian. https://www.theguardian.com/technology/2019/nov/12/googlemedical-data-project-nightingale-secret-transfer-us-health-infor mation?CMP=share_btn_tw. Accessed 20 Aug 2020

Prainsack, B., and A. Buyx. 2013. A solidarity-based approach to the governance of research biobanks. Medical Law Review 21: 71-91.

Purtova, N. 2015. The illusion of personal data as no one's property. Law, Innovation and Technology 7 (1): 83-111.

Purtova, N. 2017. Health data for common good: Defining the boundaries and social dilemmas of data commons. In Under observation: The interplay between eHealth and surveillance. Law, Governance and Technology Series. Issues in Privacy and Data Protection, vol. 35, ed. S. Adams, N. Purtova, and R. Leenes, 177-210. Cham: Springer.

Riordan, F., C. Papoutsi, J.E. Reed, C. Marston, D. Bell, and A. Majeed. 2015. Patient and public attitudes towards informed consent models and levels of awareness of Electronic Health Records 
in the UK. International Journal of Medical Informatics 84 (4): 237-247.

Rodwin, M.A. 2009. The case for public ownership of patient data. JAMA 302 (1): 86-88. https://doi.org/10.1001/jama.2009.965.

Rumbold, J.M., and B. Pierscionek. 2017. The effect of the general data protection regulation on medical research. Journal of Medical Internet Research 19 (2): e47.

Shelton, R.H. 2011. Electronic consent channels: preserving patient privacy without handcuffing researchers. Science Translational Medicine 3 (69): $69 \mathrm{~cm} 4$.

Simon, G.E., G. Coronado, L.L. DeBar, L.M. Dember, B.B. Green, S.S. Huang, et al. 2017. Data sharing and embedded research. Annals of Internal Medicine 167 (9): 668-670.

Simon, G.E., S.M. Shortreed, R.Y. Coley, R.B. Penfold, R.C. Rossom, B.E. Waitzfelder, K. Sanchez, and F.L. Lynch. 2019. Assessing and minimizing re-identification risk in research data derived from health care records. eGEMs (generation Evidence \& Methods to Improve Patient Outcomes) 7 (1): 6.

Skovgaard, L. L., Wadmann, S., and K. Hoeyer. 2019. A review of attitudes towards the reuse of health data among people in the European Union: The primacy of purpose and the common good. Health policy 123(6): 564-571.

Stevenson, F. 2015. The use of electronic patient records for medical research: Conflicts and contradictions. BMC Health Services Research 15 (1): 124.

Stevenson, F., N. Lloyd, L. Harrington, and P. Wallace. 2012. Use of electronic patient records for research: Views of patients and staff in general practice. Family Practice 30 (2): 227-232.

Strech, D., M. Mertz, H. Knuppel, G. Neitzke, and M. Schmidhuber. 2013. The full spectrum of ethical issues in dementia care:
Systematic qualitative review. The British Journal of Psychiatry: THe Journal of Mental Science 202: 400-406.

Sweeney, L. 2000. Simple Demographics Often Identify People Uniquely, p 32. https://dataprivacylab.org/projects/identifiability/ paper1.pdf. Accessed 20 Aug 2020

Taylor, L., L. Floridi, and B. Van der Sloot, eds. 2016. Group Privacy: New Challenges of Data Technologies, 250. Cham: Springer.

Wallace, S.E., and J. Miola. 2021. Adding dynamic consent to a longitudinal cohort study: A qualitative study of EXCEED participant perspectives. BMC Medical Ethics 22 (1): 1-10.

Weitzman, E.R., L. Kaci, and K.D. Mandl. 2010. Sharing medical data for health research: The early personal health record experience. Journal of Medical Internet Research 12 (2): e14.

Weitzman, E.R., S. Kelemen, L. Kaci, and K.D. Mandl. 2012. Willingness to share personal health record data for care improvement and public health: A survey of experienced personal health record users. BMC Medical Informatics and Decision Making 12 (1): 39.

Willison, D.J., M. Swinton, L. Schwartz, J. Abelson, C. Charles, D. Northrup, and L. Thabane. 2008. Alternatives to project-specific consent for access to personal information for health research: Insights from a public dialogue. BMC Medical Ethics 9 (1): 18.

Publisher's Note Springer Nature remains neutral with regard to jurisdictional claims in published maps and institutional affiliations. 